etary Sciences expressed deep concern that no work was being undertaken to identify and avoid pollution problems in interplanetary space. Commission 22 set up small a working group with I P Williams as convenor. It identified several problems requiring further study and recommended further investigation.

At the 22nd General Assembly in The Hague in 1994 a formal Working Group on The Prevention of Interplanetary Pollution was set up with members from each of the Commissions in the Division of Planetary Sciences. Care was taken to include members from each of the international powers with a presence in space operations and research. Astronomers from Australia, China P R, the Czech Republic, France, Japan, the Russian Confederation and the United States of America. Largely through e-mail contact the convenor, C S L Keay, drew on the expertise among the nominated members to expand the earlier report.

The principal areas of concern dealt with by the Group are:

* Environmentally harmful propellant residues;

* Unconfined debris from impacting objects;

* Pollution from explosives, particularly nuclear;

* Radionuclide pollution from nuclear power generators;

* Undesirable transfers of surface materials;

* Biocontamination prevention and quarantine measures.

It was stessed that the challenge is to devise cost-effective techniques and procedures acceptable to mission planners which will prevent or at the very least minimise pollution outcomes. To achieve success a program of careful preliminary research will be necessary, paying attention to the following points in regard to all aspects of space missions:

* Identify likely sources of undesirable pollution;

* Measure present levels and predict future levels;

* Assess future impact of pollution on research goals;

* Investigate likely severity and irreversibility;

* Formulate preventative or minimisation procedures;

* Set guidelines for assessing preservation values;

* Develop means to protect sensitive environments;

* Seek ways to increase awareness of the issues.

The working Group recommended the establishment of an international scientific committee to involve all relevant agencies and draw up suitable procedures and protocols for achieving success. It should be very much less costly to be proactive in this endeavour than to allow permanent harm to sensitive regions of the interplanetary environment.

\title{
14. THE PROCESS OF FREQUENCY MANAGEMENT, INTERNATIONAL TREATIES AND THE RESPONSIBILITY OF ASTRONOMERS
}

\section{J. Tarter (SETI Institute)}

Working with national and international regulatory bodies to allocate the radio spectrum, and monitoring regional applications for spectrum usage are increasingly becoming the cost of doing radio astronomy. Economic incentives are enormous, with new services constantly seeking additional allocations within the radio spectrum. Radio astronomers cannot depend on the goodwill of forprofit service providers to keep portions of the spectrum free for the use of the passive services (radio astronomy and remote sensing). At present, a small number of senior scientists and managers are struggling against this tide. They should not only be praised, and thanked, but joined. It is imperative that current and future generations of radio astronomers learn the business of frequency management and participate in the distribution of this scarce resource.

\section{EDUCATING THE PUBLIC ABOUT PRESERVATION OF THE ASTRONOMICAL WINDOWS}

\section{W. T. Sullivan, III (Univ. of Washington)}

If astronomers are going to succeed in their efforts to keep the skies free of light pollution and radio interference, then they must deal not only with lighting and radio engineers, business concerns, and politicians, but also the general public. If the public becomes persuaded that clear, pristine skies are worth having for the same sorts of reasons that we value drinkable water and 